\title{
Cardinal factorization of finite relational structures
} by

\author{
C. C. Chang * (Los Ángeles, Calif.)
}

Introduction. This paper is a continuation of the work of ChangJónsson-Tarski [4]. It is also, to some extent, complementary to the to be any overlap between the as far as we know, there does not appear does not differ very much the results here and those in [5]. Our notation we try to state our results from that of [4]; in fact, whenever possible, in mind, especially results in simpler terms. This point should be kept For the purposes of we repeat the definitions given in [4].

structure, is a pair $\mathfrak{A}=\langle A, P\rangle$ paper, a relational structure, or simply a ternary relation over $\mathfrak{A}=\langle A, P\rangle$ where $A$ is a non-empty set and $P$ is of $A$, and define $\|\mathfrak{A}\|=|A|$ i.e., $P \subset A^{3}$. We let $|A|$ denote the cardinal are finite, and that they. Henceforth, we shall assume that all structures are finite, and that they are denoted by $\mathfrak{A}=\langle A, P\rangle, \mathfrak{B}=\langle B, Q\rangle$, wise, when we mention 'structs. Thus, unless specifically stated otherletters $\mathfrak{X}, \mathfrak{B}, \mathfrak{C}$ mention 'structure' we mean 'finite structure', and the that a structure $\mathfrak{O}$ is inde over finite structures. Recall from [4] then either $\|\mathfrak{B}\|=1$ or $\|\mathfrak{C}\|=1$ if $\|\mathfrak{A}\|>1$ and whenever $\mathfrak{A} \cong \mathfrak{B} \times \mathfrak{C}$ then either $\|\mathfrak{B}\|=1$ or $\|\mathfrak{C}\|=1$. If $P \neq 0$, then this also implies that indecompon sotion than indecomposable structures is that of a prime structure. We first say that a structure $\mathfrak{A}$ divides another structure $\mathfrak{B}$, in symbols $\mathfrak{A} \| \mathfrak{B}$, if there is $\mathfrak{X} \| \mathfrak{B} \times \mathfrak{C}$ that $\mathfrak{U} \times \mathfrak{C} \cong \mathfrak{B}$. A structure $\mathfrak{A}$ is prime if whenever $\mathfrak{A} \| \mathfrak{B} \times \mathfrak{C}$ then either $\mathfrak{A} \| \mathfrak{B}$ or $\mathfrak{A} \| \mathfrak{C}$. If $\|\mathfrak{U}\|>1$ and $\mathfrak{A}$ is prime, then $\mathfrak{A}$ is indecomposable. The structure $\mathbf{1}=\langle\{0\},\{\langle 0,0,0\rangle\}\rangle$ is prime but not indecomposable, and there are also examples of indecomposable but non-prime structures. A structure $\mathfrak{A}$ is cancelable if $\mathfrak{A} \times \mathfrak{B} \cong \mathfrak{U} \times \mathfrak{C}$ implies $\mathfrak{B} \cong \mathbb{C}$. Notice that the notions of being prime or cancelable depend very

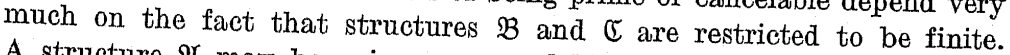
A structure $\mathfrak{A}$ may be prime or cancelable in one sense without being prime or cancelable if $\mathfrak{B}$ and $\mathfrak{C}$ are allowed to range over infinite
structures.

* Research supported in part by NSF Grant GP 5600. 
One of the main results of [4], when stated in our simplified language, is the following:

(I) Let $\mathfrak{A}$ be a structure. Suppose there are a binary reflexive connected relation $S \in \Delta(\mathfrak{U})$ whose field is $A$, and an element $a \in A$ such that

(i) $a S x$ and $x S a$ imply $a=x$ for all $x \in A$, and

(ii) $P(a, a, a)$, then $\mathfrak{A}$ has the unique factorization property.

The main improvement offered in this paper is the following: (Theorem 2.8)

(II) Let $\mathfrak{A}$ be a structure and let $S \in \Delta(\mathfrak{X})$ be a binary reflexive connected relation whose field is $A$. Let $T$ be the least equivalence relation over $A$ containing $S \cap \breve{S}$. If $a$ is any element of $A$ such that

(i) the substructure

$$
\mathfrak{A}(T, a)=\left\langle a \mid T, P \cap(a / T)^{3}\right\rangle
$$

is a cardinal product of prime and cancelable factors, and (ii) $P(a, a, a)$, then $\mathfrak{U}$ has the unique factorization property. Observe that under the hypotheses of $(\mathrm{I})$, the substructure

$$
\mathfrak{U}(T, a)=\langle\{a\},\{\langle a, a, a\rangle\}\rangle \cong \mathbf{1}
$$

is certainly a product of prime and cancelable factors. Actually the condition (i) of (II), namely that $\mathfrak{A}(T, a)$ be a product of prime and cancelable factors can be weakened somewhat if the structure of $\mathfrak{A}(T, a)$ is particularly simple, see Theorems 2.1 and 2.2. The interesting and important open problem is whether the condition (i) in (II) can, in general, be weakened to

(i') the substructure $\mathfrak{A}(T, a)$ has the unique factorization property.

If this is the case, then a possible inductive proof might be given for the proposition that every structure $\mathfrak{A}$ with a binary reflexive connected $S \in \Delta(\mathfrak{H})$ whose field is $A$ has the unique factorization property.

This is the extent of our results for structures. The biggest remaining problem seems to be finding useful examples of (product of) prime and cancelable structures. It is almost unnecessary to point out that these results hold for structures with possibly many finitary relations; one only has to reformulate the notions of indecomposable, prime, and cancelable for the new classes of finite structures.

It turns out that for the class of structures $\mathfrak{A}=\langle A, R\rangle$ where $R$ is a binary reflexive relation whose field is $A$ the specialization of our general results leads to some new and concrete results for binary reflexive relations. In what follows, instead of the pairs $\langle A, R\rangle$, we shall only consider non-empty binary reflexive relations $R, s, T, \ldots$, etc., again they are assumed to be finite. The specialization of (I) to this case was stated in [4] (and announced in [1]) as follows:

(I') Let $R$ be a connected relation with at least one element $a$ in its field such that

(i) $x R a$ and $a R x$ imply $a=x$ for all $x \in \operatorname{Fld}(R)$, then $R$ has the unique factorization property.

The analog of (II) is the following:

(II') Let $R$ be a connected relation and let $T$ be the least equivalence relation containing $R \cap \breve{R}$. If there is an element $a \in \operatorname{Fld}(R)$ such that (i) the subrelation

$$
R(T, a)=R \cap(a / T)^{2}
$$

is a product of prime and cancelable (in the sense for relations factors,

then $R$ has the unique factorization property.

Note again that if $a$ is an anti-symmetric element of $R$ then $R(T, a)$ $=\{\langle a, a\rangle\}$ which is prime and cancelable. Just as in the case of (II), condition (i) of ( $\left.\mathrm{II}^{\prime}\right)$ may be weakened if $R(T, a)$ has a simple structure; see the discussion before Theorem 2.9. The interesting thing is that in this particular special case of relations, we are able to prore that: (Theorem 2.9)

(III) Every (finite) square relation (i.e. $R=$ Fld $(R)^{2}$ ) is a product of prime and cancelable factors.

One can now combine (III) with $\left(\mathrm{II}^{\prime}\right)$. As a consequence we get: (Theorem 2.16)

(IV) Every transitive connected relation $R$ has the unique factorization property.

This sums up all of our results in this paper. The methods, especially for those results in Section 1 holding for structures in general, are largely extensions of the methods of [4]. On the other hand, since our methods do not allow us to prove that a structure $\mathfrak{A}$ has the strict or the intermediate refinement property (see [4] for definition), they are a departure from and a step beyond the methods used in [4]. Section 1 is concerned with what we have called a central refinement theorem (Theorem 1.3), and Section 2 points out the main consequences of this theorem. As far as we know, most of our results have modifications so that they will hold even if we consider infinite structures. The pleasure of discovering what these modifications are is reserved for the reader.

§ 1. A central refinement theorem. We begin by recalling some notions from [4], always remembering that we are dealing with a simplified situation. 
Suppose that $E$ is an equivalence relation over the set $A$ of the structure $\mathfrak{U}=\langle A, P\rangle$. As usual $a / E$ denotes the $E$-equivalence class given by $a \in A$, and $A / E$ the set of all such equivalence classes, i.e.,

$$
A / E=\{a / E: a \in A\} \text {. }
$$

A natural ternary relation $P^{*} E$ on $A$ given by $P$ and $E$ is defined as follows:

$P^{*} E=\{\langle a, b, c\rangle:$ there exist $x, y, z$ such that

$$
P(x, y, z) \text { and } x E a, y E b, z E c\} .
$$

In terms of $P^{*} E$, we define

$$
P / E=\left\{\langle a / E, b / E, c / E\rangle: P^{*} E(a, b, c)\right\},
$$

and the quotient structure

$$
\mathfrak{U} / E=\langle A / E, P / E\rangle .
$$

The dual of the quotient structures are the substructures

$$
\mathfrak{U}(E, a)=\left\langle a / E, P \cap(a / E)^{3}\right\rangle
$$

which we already mentioned in the introduction. We say that the relations $R, R^{\prime}$ is a pair of factor relations of $E$ over $\mathfrak{A}$ if

(i) $R, R^{\prime}$ are equivalence relations over $A$;

(ii) $R \cap R^{\prime}=E$;

(iii) $R \mid R^{\prime}=A^{2}$

(iv) $P^{*} R \cap P^{*} R^{\prime}=P^{*} E$.

In case $E$ is the identity relation on $A$, we simply say that $R, R^{\prime}$ is a pair of factor relations over $\mathfrak{A}$. It is shown in [4] that: $\mathfrak{X} / \mathbb{E} \cong \mathfrak{B} \times \mathfrak{C}$ if and only if there is a pair of factor relations $R, R^{\prime}$ of $E$ over $\mathfrak{A}$ such that $\mathfrak{U} / R \cong \mathfrak{B}$ and $\mathfrak{U} / R^{\prime} \cong \mathfrak{C}$. If there is an $a \in A$ such that $P(a, a, a)$, then we can equivalently say that

$$
\mathfrak{A}\left(R^{\prime}, a\right) \cong \mathfrak{B} \cong \mathfrak{A} / R \quad \text { and } \quad \mathfrak{A}(R, a) \cong \mathfrak{C} \cong \mathfrak{A} / R^{\prime} .
$$

We now introduce a slightly different notion. A pair of relations $R, R^{\prime}$ is said to be orthogonal over $\mathfrak{A}$ and $E$ if

(i) $R, R^{\prime}$ are equivalence relations over $A$;

(ii) $R \cap R^{\prime}$ is the identity on $A$;

(iii) $R \mid R^{\prime}=E$;

(iv) $P^{*} R \cap P^{*} R^{\prime}=P$.

In the same way that the substructure $\mathfrak{A}(R, a)$ is dual to the quotient structure $\mathfrak{U} / R$, this notion of being orthogonal is dual to being factor relations. For instance it is immediately seen that $R, R^{\prime}$ is a pair of factor

relations of the identity over $\mathfrak{A}$ if and only if $R, R^{\prime}$ are orthogonal over $\mathfrak{A}$ and $A^{2}$.

LEMv's 1.1. Suppose that $R, R^{\prime}$ are orthogonal over $\mathfrak{A}$ and $E$, and let $a \in A$. Then the relations $R \cap(a / E)^{2}, R^{\prime} \cap(a / E)^{2}$ is a pair of factor relations over $\mathfrak{A}(E, a)$. In particular, if $P(a, a, a)$, then

$$
\mathfrak{A}(H, a) \cong \mathfrak{A}(R, a) \times A\left(R^{\prime}, a\right) .
$$

Proof. Let $B=a / E, Q=P \cap B^{3}, \mathfrak{B}=\langle B, Q\rangle, S=R \cap B^{2}$, and $S^{\prime}=R^{\prime} \cap B^{2}$. It is a simple matter to check that:

(i) $S, S^{\prime}$ are equivalence relations over $B$;

(ii) $S \cap S^{\prime}$ is the identity on $B$;

(iii) $S \mid S^{\prime}=B^{2}$

(iv) $Q^{*} S \cap Q^{*} S^{\prime}=Q$

So our first conclusion is verified, and we get

$$
\mathfrak{A}(E, a)=\mathfrak{B} \cong \mathfrak{B} / \mathbb{S} \times \mathfrak{B} / \mathbb{S}^{\prime}
$$

In the case where $P(a, a, a)$, we get

$$
\mathfrak{B} \cong \mathfrak{B}(S, a) \times \mathfrak{B}\left(S^{\prime}, a\right) .
$$

Since $R$ and $R^{\prime}$ are subsets of $E$, we have

$$
\mathfrak{B}(S, a)=\mathfrak{A}(R, a) \quad \text { and } \quad \mathfrak{B}\left(S^{\prime}, a\right)=\mathfrak{A}\left(R^{\prime}, a\right) .
$$

The lemma is proved.

We now remind the reader to look up the definition of the set $\Delta(\mathfrak{U})$ in [4], again remember to simplify.

LEMMA 1.2. Suppose that $R, R^{\prime}$ and $S, S^{\prime}$ are two pairs of factor relations over $\mathfrak{A}$, and suppose that $T \in \Delta(\mathfrak{H})$ is a binary reflexive connected relation whose field is $A$. Let $U$ be the least equivalence relation containing $T \cap \breve{T}$. Then

(a) the pair $R \cap U, R^{\prime} \cap U$ is orthogonal over $\mathfrak{A}$ and $U$;

(b) the pair $R, R^{\prime} \cap U$ is orthogonal over $\mathfrak{A}$ and $R \mid U$;

(c) the pair $(R \mid U) \cap S,(R \mid U) \cap S^{\prime}$ is orthogonal over $\mathfrak{A}$ and $R \mid U$;

(d) the pair $(R \mid U) \cap S, U \cap S^{\prime}$ is orthogonal over $\mathfrak{A}$ and $(R \mid U) \cap(S \mid U)$.

Proof. For each one the assertions (a)-(d), we shall check that the conditions (i)-(iv) of the definition of orthogonality are satisfied.

Let us dismiss condition (iv) for all the cases first. Notice that for every pair of relations $Q, Q^{\prime}$ given in (a)-(d), we either have $Q \subset R$ and $Q^{\prime} \subset R^{\prime}$ or else $Q \subset S$ and $Q^{\prime} \subset S^{\prime}$. Since both $Q$ and $Q^{\prime}$ are at least reflexive, we have immediately that

$$
P^{*} Q \cap P^{*} Q^{\prime}=P
$$


Next, by exactly the same observation we see that every pair $Q, Q^{\prime}$ given in (a)-(d) satisfies

$$
Q \cap Q^{\prime} \text { is the identity on } A \text {. }
$$

So condition (ii) can also be dismissed.

Referring back to the definition of $P^{*} E$, we see that this notion can be extended in a natural way to any $n$-placed relation $W$ and any equivalence relation $E$ to get the set $W^{*} E$. By a straightforward induction based on the definition of $\Delta(\mathfrak{U})$, we can prove that

$$
W^{*} R \cap W^{*} R^{\prime}=W \quad \text { for all } W^{\prime} \in \Delta(\mathfrak{2}) .
$$

We shall not carry out the details of this argument, but only point out that the hypothesis that $R, R^{\prime}$ be a pair of factor relations over $\mathfrak{A}$ must be used. In case $W$ is a binary relation in $\Delta(\mathfrak{L})$, then (1) may be rewritten in terms of the more familiar relative product symbol | as follows:

$$
[R|W| R] \cap\left[R^{\prime}|W| R^{\prime}\right]=W .
$$

By hypothesis, $T \in \Delta(\mathfrak{U})$, whence $\breve{T} \in \Delta(\mathfrak{R}),(T \cap \breve{T}) \in \Delta(\mathfrak{U})$, and, in fact, the relation

$$
(T \cap \breve{T})^{n}=(T \cap \breve{T})|(T \cap \breve{T})| \ldots \mid(T \cap \breve{T}) n \text {-times }
$$

belongs to $d(\mathfrak{L})$ for every $n$. Thus we see from (2) that

(3) $\quad\left[R\left|(T \cap \breve{T})^{n}\right| R\right] \cap\left[R^{\prime}\left|(T \cup \breve{T})^{n}\right| R^{\prime}\right]=(T \cap \breve{T})^{n} \quad$ for every $n$.

By definition, $U=\bigcup_{n}(T \cap \breve{T})^{n}$, so a simple argument from (3) shows that

$$
[R|U| R] \cap\left[R^{\prime}|U| R^{\prime}\right]=U .
$$

Let us now go back and finish case (a). It is clear that $R \cap U, R^{\prime} \cap U$ are equivalence relations over $A$. It is equally clear that

$$
(R \cap U) \mid\left(R^{\prime} \cap U\right) \subset U \text {; }
$$

so it only remains to prove that

$$
U \subset(R \cap U) \mid\left(R^{\prime} \cap U\right) .
$$

Suppose that $a U b$. We know that there is an element $c$ such that $a R c R^{\prime} b$. Using the reflexivity of all the relations involved, we have

$$
a(R|U| R) c \quad \text { and } \quad a\left(R^{\prime}|U| R^{\prime}\right) c .
$$

Whence, by (4), aUc. Similarly $e U b$, so

This proves (a).

$$
a(R \cap U) c\left(R^{\prime} \cap U\right) b .
$$

For (b), we need first to show that $R \mid U$ is an equivalence relation over $A$. It is sufficient to show that $R$ and $U$ permute, that is

$$
R|U=U| R .
$$

So suppose $a(R \mid U) b$. There is a $c$ such that $a R^{\prime} c R b$. Whence

$$
a(R|U| R) c \quad \text { and } \quad a\left(R^{\prime}|U| R^{\prime}\right) c,
$$

and, by (4), $a U c$, so $a(U \mid R) b$. This proves $R|U \subset U| R$, and symmetry does the rest. To finish case (b), we show that

$$
R\left|\left(R^{\prime} \cap U\right)=R\right| U \text {. }
$$

Again, one inclusion is obvious. Let $a(R \mid U) b$. There is a $c$ such that $a R c R^{\prime} b$. Now

$$
c(R|U| R) b \quad \text { and } \quad c\left(R^{\prime}|U| R^{\prime}\right) b,
$$

so that $c U b$, and this gives $a\left(R \mid\left(R^{\prime} \cap U\right)\right) b$.

Continuing with (c), it is evident by (5) that $(R \mid U) \cap S,(R \mid U) \cap S^{\prime}$ are both equivalence relations over $A$. We now have to show the equality

$$
((R \mid U) \cap S)\left|\left((R \mid U) \cap S^{\prime}\right)=R\right| U \text {. }
$$

As before, we only need to show one inclusion, that from right to left. We point out that here is where we need that $T$ is connected. Suppose $a(R \mid D) b$. There is a $c$ such that $a S c S^{\prime} b$. Let $f$ and $g$ be the decomposition functions for the pairs $S^{\prime}, S^{\prime}$ and $R, R^{\prime}$, respectively. That is, for all $x, y \in A$,

and

$$
f(x, y) \text { is the unique } z \in A \text { such that } x S z S^{\prime} y \text {, }
$$

$$
g(x, y) \text { is the unique } z \in A \text { such that } x R z R^{\prime} y \text {. }
$$

See [4], or [5], for some of the elementary properties of decomposition functions. Recall from the proof of Theorem 7.3 in [4] that (reading $T$ for $S$ and $U$ for $\equiv)$ :

$$
f_{x} g_{x}(y) U g_{x} f_{x}(y), \quad \text { for all } x, y \in A \text {. }
$$

Let $d$ be such that $a R d U b$. Using the elementary properties of decomposition functions and (6), we have

$$
c=g_{c} f_{c}(c)=g_{c} f_{c}(a) U f_{c} g_{c}(a)=f_{c} g_{c}(d) .
$$

Using (4), the functions $f$ and $g$ preserve $\dot{U}$, whence

$$
f_{c} g_{c}(d) U f_{c} g_{c}(b) U g_{c} f_{c}(b)=g_{c} f_{b}(b)=g_{c}(b) R b .
$$

So $c(U \mid R) b$, and by $(5), c(R \mid U) b$. In an entirely analogous manner, we have $a(R \mid U) c$, whence $\left.a((R \mid U) \cap S) \mid(R \mid U) \cap S^{\prime}\right) b$, as to be shown. 
Finally, to finish case (d), we only need to prove the equality

$$
((R \mid U) \cap S) \mid\left(U \cap S^{\prime}\right)=(R \mid U) \frown(S \mid U) .
$$

Now the inclusion from left to right follows from straightforward calculations. To go the other way, suppose that

$$
\langle a, b\rangle \epsilon(R \mid U) \cap(S \mid U) .
$$

Since this already implies $a(R \mid U) b$, by the results of (c), we know that there is a $c$ such that

$$
a((R \mid U) \cap S) c\left((R \mid U) \cap S^{\prime}\right) b .
$$

Because $a(S \mid U) b$, we see that

$$
c S^{\prime}|U| S^{\prime} b \quad \text { and } \quad c S|U| S b,
$$

whence, by (5), $c U b$, thus

and

$$
a((R \mid U) \cap S) c\left(U \cap S^{\prime}\right) b
$$

$$
\langle a, b\rangle \epsilon((R \mid U) \cap S) \mid\left(U \cap S^{\prime}\right) .
$$

This concludes the proof of the lemma.

Suppose that $T \in \Delta(\mathfrak{H})$ is a binary reflexive connected relation over $A, U$ is the least equivalence relation containing $T \frown \breve{T}$, and $P(a, a, a)$. We shall call the substructure $\mathfrak{U}(U, a)$ a center of $\mathfrak{A}$. Conversely, any substructure $\mathfrak{A}^{\prime}$ of $\mathfrak{A}$ is a center of $\mathfrak{A}$ if there are appropriate $T, U, a$ such that $\mathfrak{U}^{\prime}=\mathfrak{H}(U, a)$.

THEOREM 1.3. (CENTRAL REFINEMENT THEOREM.) Suppose that $\mathfrak{A} \cong \mathfrak{B}_{1} \times \mathfrak{B}_{2} \cong \mathfrak{C}_{1} \times \mathfrak{C}_{2}$ and suppose that $\mathfrak{X}^{\prime}$ is any center of $\mathfrak{A}$. Then the following hold:

(i) There are $\mathfrak{B}_{1}^{\prime}, \mathfrak{B}_{2}^{\prime}, \mathfrak{C}_{1}^{\prime}, \mathfrak{C}_{2}^{\prime}$ such that

$$
\mathfrak{A}^{\prime} \cong \mathfrak{B}_{1}^{\prime} \times \mathfrak{B}_{2}^{\prime} \cong \mathfrak{C}_{1}^{\prime} \times \mathbb{C}_{2}^{\prime} .
$$

(ii) There are $\mathfrak{B}_{11}, \mathfrak{B}_{12}, \mathfrak{B}_{21}, \mathfrak{B}_{22}$ such that

$$
\begin{aligned}
& \mathfrak{B}_{1} \times \mathfrak{B}_{1}^{\prime} \cong \mathfrak{B}_{11} \times \mathfrak{B}_{12}, \\
& \mathfrak{B}_{2} \times \mathfrak{B}_{2}^{\prime} \cong \mathfrak{B}_{21} \times \mathfrak{B}_{22} .
\end{aligned}
$$

(iii) There are $\mathfrak{F}_{11}, \mathfrak{C}_{12}, \mathfrak{C}_{21}, \mathfrak{V}_{22}$ such that

$$
\begin{aligned}
& \mathfrak{c}_{1} \times \mathbb{C}_{1}^{\prime} \cong \mathfrak{c}_{11} \times \mathfrak{c}_{12}, \\
& \mathfrak{c}_{2} \times \mathfrak{c}_{2}^{\prime} \cong \mathfrak{c}_{21} \times \mathfrak{c}_{22} .
\end{aligned}
$$

(iv) Finally, the following additional relations hold:

$$
\begin{aligned}
& \mathfrak{C}_{1}^{\prime} \times \mathfrak{B}_{11} \cong \mathfrak{B}_{1}^{\prime} \times \mathfrak{C}_{11}, \\
& \mathfrak{C}_{1}^{\prime} \times \mathfrak{B}_{21} \cong \mathfrak{B}_{2}^{\prime} \times \mathfrak{C}_{12}, \\
& \mathbb{C}_{2}^{\prime} \times \mathfrak{B}_{12} \cong \mathfrak{B}_{1}^{\prime} \times \mathfrak{C}_{21}, \\
& \mathfrak{C}_{2}^{\prime} \times \mathfrak{B}_{22} \cong \mathfrak{B}_{2}^{\prime} \times \mathfrak{C}_{22} .
\end{aligned}
$$

Proof. We may suppose that $\mathfrak{X}^{\prime}=\mathfrak{A}(U, a)$ for some appropriate $T, U, a$. Let $R, R^{\prime}$ and $S, S^{\prime}$ be pairs of factor relations over $\mathfrak{A}$ such that

$$
\begin{aligned}
\mathfrak{B}_{1} \cong \mathfrak{H}(R, a), & \mathfrak{B}_{2} \cong \mathfrak{A}\left(R^{\prime}, a\right), \\
\mathfrak{C}_{1} \cong \mathfrak{A}(S, a), & \mathfrak{C}_{2} \cong \mathfrak{A}\left(S^{\prime}, a\right) .
\end{aligned}
$$

Reading off the orthogonal relations in Lemma 1.2, remembering that we may switch the roles of $R, R^{\prime}$ and $S, S^{\prime}$ freely, we get, by Lemma 1.1, the following:

$$
\begin{aligned}
& \mathfrak{U}(U, a) \cong \mathfrak{A}(R \cap U, a) \times \mathfrak{A}\left(R^{\prime} \cap U, a\right) \\
& \cong \mathfrak{A}(S \cap U, a) \times \mathfrak{A}\left(S^{\prime} \cap U, a\right) .
\end{aligned}
$$

$\mathfrak{U}(R \mid U, a) \cong \mathfrak{A}((R \mid U) \cap S, a) \times \mathfrak{H}\left((R \mid U) \cap S^{\prime}, a\right)$.

$\mathfrak{U}\left(R^{\prime} \mid U, a\right) \cong \mathfrak{U}\left(\left(R^{\prime} \mid U\right) \cap S, a\right) \times \mathfrak{A}\left(\left(R^{\prime} \mid U\right) \cap S^{\prime}, a\right)$.

$\mathfrak{U}(S \mid U, a) \cong \mathfrak{I}((S \mid U) \cap R, a) \times \mathfrak{H}\left((S \mid U) \cap R^{\prime}, a\right)$.

$\mathfrak{U}\left(S^{\prime} \mid U, a\right) \cong \mathfrak{A}\left(\left(S^{\prime} \mid U\right) \cap R, a\right) \times \mathfrak{A}\left(\left(S^{\prime} \mid U\right) \cap R^{\prime}, a\right)$.

$$
\begin{aligned}
& \mathfrak{U}((R \mid U) \cap(S \mid U), a) \cong \mathfrak{A}((R \mid U) \cap S, a) \times \mathfrak{A}\left(U \cap S^{\prime}, a\right) \\
& \cong \mathfrak{A}((S \mid U) \cap R, a) \times \mathfrak{A}\left(U \cap R^{\prime}, a\right) . \\
& \mathfrak{A}\left(\left(R^{\prime} \mid U\right) \cap(S \mid U), a\right) \cong \mathfrak{A}\left(\left(R^{\prime} \mid U\right) \cap S, a\right) \times \mathfrak{H}\left(U \cap S^{\prime}, a\right) \\
& \cong \mathfrak{H}\left((S \mid U) \cap R^{\prime}, a\right) \times \mathfrak{A}(U \cap R, a) . \\
& \mathfrak{U}\left(\left(R \mid U \cap\left(S^{\prime} \mid U\right), a\right) \cong \mathfrak{A}\left((R \mid U) \cap S^{\prime}, a\right) \times \mathfrak{A}(U \cap S, a)\right. \\
& \cong \mathfrak{A}\left(\left(S^{\prime} \mid U\right) \cap R, a\right) \times \mathfrak{U}\left(U \cap R^{\prime}, a\right) . \\
& \mathfrak{U}\left(\left(R^{\prime} \mid U\right) \cap\left(S^{\prime} \mid U\right), a\right) \cong \mathfrak{U}\left(\left(R^{\prime} \mid \bar{U}\right) \cap S^{\prime}, a\right) \times \mathfrak{A}(U \cap S, a) \\
& \cong \mathfrak{U}\left(\left(S^{\prime} \mid U\right) \cap R^{\prime}, a\right) \times \mathfrak{U}(U \cap R, a) .
\end{aligned}
$$


It now only remains to define:

$$
\begin{aligned}
& \mathfrak{B}_{1}^{\prime}=\mathfrak{U}\left(R^{\prime} \cap U, a\right), \quad \mathfrak{B}_{2}^{\prime}=\mathfrak{H}(R \cap U, a), \\
& \mathfrak{C}_{1}^{\prime}=\mathfrak{H}\left(S^{\prime} \cap U, a\right), \quad \mathbb{C}_{2}^{\prime}=\mathfrak{H}\left(S^{\prime} \cap U, a\right) . \\
& \mathfrak{B}_{11}=\mathfrak{A}((R \mid U) \cap S, a), \quad \mathfrak{B}_{12}=\mathfrak{H}\left((R \mid U) \cap S^{\prime}, a\right), \\
& \mathfrak{B}_{21}=\mathfrak{A}\left(\left(R^{\prime} \mid U\right) \cap S^{\prime}, a\right), \quad \mathfrak{B}_{22}=\mathfrak{A}\left(\left(R^{\prime} \mid U\right) \cap S^{\prime}, a\right) . \\
& \mathbb{C}_{11}=\mathfrak{A}((S \mid U) \cap R, a), \quad \mathbb{C}_{12}=\mathfrak{A}\left((S \mid U) \cap R^{\prime}, a\right), \\
& \mathbb{C}_{21}=\mathfrak{A}\left(\left(S^{\prime} \mid U\right) \cap R, a\right), \quad \mathbb{C}_{22}=\mathfrak{A}\left(\left(S^{\prime} \mid U\right) \cap R^{\prime}, a\right) .
\end{aligned}
$$

Now, conclusion (i) follows from (a'), conclusions (ii) and (iii) follow from $\left(b^{\prime}\right)$ and $\left(c^{\prime}\right)$, and conclusion (iv) follows from $\left(d^{\prime}\right)$. The theorem is proved.

§2. Applications. In this section we are concerned with the generalizations of $(\mathrm{I})$ and $\left(\mathrm{I}^{\prime}\right)$ mentioned in the introduction.

Recall that a structure $\mathfrak{A}$ has the refinement property if whenever

$$
\mathfrak{U} \cong \mathfrak{B}_{1} \times \mathfrak{B}_{2} \cong \mathfrak{C}_{1} \times \mathfrak{C}_{2},
$$

then there are $\mathfrak{D}_{11}, \mathfrak{D}_{12}, \mathfrak{D}_{21}, \mathfrak{D}_{22}$ such that

$$
\begin{aligned}
& \mathfrak{B}_{1} \cong \mathfrak{D}_{11} \times \mathfrak{D}_{12}, \quad \mathfrak{B}_{2} \cong \mathfrak{D}_{21} \times \mathfrak{D}_{22}, \\
& \mathfrak{C}_{1} \cong \mathfrak{D}_{11} \times \mathfrak{D}_{21}, \quad \mathbb{C}_{2} \cong \mathfrak{D}_{12} \times \mathfrak{D}_{22} .
\end{aligned}
$$

In this case we say that the structure $\mathfrak{D}_{11}, \mathfrak{D}_{12}, \mathfrak{D}_{21}, \mathfrak{D}_{22}$ is a refinement of $\mathfrak{B}_{1}, \mathfrak{B}_{2}, \mathfrak{C}_{1}, \mathfrak{C}_{2}$. So, $\mathfrak{U}$ has the refinement property if and.only if whenever $\mathfrak{A} \cong \mathfrak{B}_{1} \times \mathfrak{B}_{2} \cong \mathfrak{C}_{1} \times \mathfrak{C}_{2}$, then there exists a refinement of $\mathfrak{B}_{1}, \mathfrak{B}_{2}$, $\mathfrak{V}_{1}, \mathfrak{C}_{2}$. A finite structure has a unique factorization into indecomposable factors if and only if it has the refinement property.

THEOREM 2.1. Suppose that some center of $\mathfrak{A}$ is indecomposable and cancelable, then $\mathfrak{a}$ has the refinement property.

Proof. Let $\mathfrak{A}^{\prime}$ be a center of $\mathfrak{A}$ which is indecomposable and cancelable, and suppose that

$$
\mathfrak{A} \cong \mathfrak{B}_{1} \times \mathfrak{B}_{2} \cong \mathfrak{C}_{1} \times \mathfrak{c}_{2} .
$$

By Theorem 1.3, all of the structures listed therein exist. Since $\mathfrak{I}^{\prime}$ is indecomposable, it follows that (since the relation $P$ is never empty on a center):

either

or

$$
\mathfrak{A}^{\prime} \cong \mathfrak{B}_{1}^{\prime} \quad \text { and } \quad \mathbf{1} \cong \mathfrak{B}_{2}^{\prime},
$$

$$
\mathbf{1} \cong \mathfrak{B}_{1}^{\prime} \quad \text { and } \quad \mathfrak{U}^{\prime} \cong \mathfrak{B}_{2}^{\prime} .
$$

Similarly for $\widetilde{\mho}_{1}^{\prime}$ and $\mathbb{C}_{2}^{\prime}$. By symmetry, it is sufficient to consider the following two cases.

Case 1. $\mathfrak{A}^{\prime} \cong \mathfrak{B}_{1}^{\prime} \cong \mathbb{C}_{1}^{\prime}$ and $\mathbf{1} \cong \mathfrak{B}_{2}^{\prime} \cong \mathbb{T}_{2}^{\prime}$. In this case, by using the cancellation property of $\mathfrak{H}^{\prime}$ and 1.3 (iv), we have

$$
\begin{gathered}
\mathfrak{B}_{11} \cong \mathfrak{C}_{11}, \\
\mathfrak{C}_{1}^{\prime} \times \mathfrak{B}_{21} \cong \mathfrak{C}_{12}, \\
\mathfrak{B}_{12} \cong \mathfrak{B}_{1}^{\prime} \times \mathfrak{C}_{21}, \\
\mathfrak{B}_{22} \cong \mathfrak{C}_{22} .
\end{gathered}
$$

Putting these relations back into 1.3 (ii) and 1.3 (iii), and again cancelling $\mathfrak{U}^{\prime}$, we get the refinement

$$
\begin{aligned}
& \mathfrak{B}_{1} \cong \mathfrak{B}_{11} \times \mathfrak{C}_{21}, \quad \mathfrak{B}_{2} \cong \mathfrak{B}_{21} \times \mathfrak{C}_{22}, \\
& \mathfrak{C}_{1} \cong \mathfrak{B}_{11} \times \mathfrak{B}_{21}, \quad \mathfrak{C}_{2} \cong \mathfrak{C}_{21} \times \mathfrak{C}_{22} .
\end{aligned}
$$

Case 2. $\mathfrak{X}^{\prime} \cong \mathfrak{B}_{1}^{\prime} \cong \mathbb{C}_{2}^{\prime}$ and $\mathbf{1} \cong \mathfrak{B}_{2}^{\prime} \cong \mathbb{C}_{1}^{\prime}$. By analogous arguments, we first get

$$
\begin{gathered}
\mathfrak{B}_{11} \cong \mathfrak{B}_{1}^{\prime} \times \mathfrak{C}_{11}, \\
\mathfrak{B}_{21} \cong \mathfrak{C}_{12}, \\
\mathfrak{B}_{12} \cong \mathfrak{C}_{21}, \\
\mathfrak{C}_{2}^{\prime} \times \mathfrak{B}_{22} \cong \mathfrak{C}_{22} .
\end{gathered}
$$

Then we have the refinement

$$
\begin{aligned}
& \mathfrak{B}_{1} \cong \mathfrak{C}_{11} \times \mathfrak{B}_{12}, \mathfrak{B}_{2} \cong \mathfrak{B}_{21} \times \mathfrak{B}_{22}, \\
& \mathfrak{C}_{1} \cong \mathfrak{T}_{11} \times \mathfrak{B}_{21}, \quad \mathfrak{C}_{2} \cong \mathfrak{B}_{12} \times \mathfrak{B}_{22} .
\end{aligned}
$$

The theorem is proved.

Notice that in order for the proof to go through we only needed the existence of a structure $\mathfrak{A}^{\prime}$, not necessarily a center of $\mathfrak{A}$, for which the hypotheses of 2.1 and the conclusions of 1.3 hold. This observation is the first step of the next theorem.

THEOREM 2.2. Let $\mathfrak{A}^{\prime}$ be a center of $\mathfrak{U}$. Suppose that

(i) $\mathfrak{I}^{\prime}$ has a unique (up to isomorphism) representation

$$
\mathfrak{A}^{\prime} \cong \mathfrak{D}_{1} \times \ldots \times \mathfrak{D}_{m}
$$

as a product of indecomposable factors $\mathfrak{D}_{i}$,
(ii) each $\mathfrak{D}_{i}$ is cancelable,
(iii) $\mathfrak{D}_{i} \neq \mathfrak{D}_{j}$ if $i \neq j$, and
(iv) whenever $\mathfrak{D}_{i} \times \mathfrak{B} \cong \mathfrak{D}_{j} \times \mathfrak{C}, i \neq j$, then $\mathfrak{D}_{i} \| \mathfrak{C}$ and $\mathfrak{D}_{j} \| \mathfrak{B}$.
Then $\mathfrak{A}$ has the refinement property. 
Proof. Suppose that $\mathfrak{A} \cong \mathfrak{B}_{1} \times \mathfrak{B}_{2} \cong \mathfrak{C}_{1} \times \mathfrak{C}_{2}$. We may as well assume that $m>1$, for otherwise Theorem 2.1 will apply. We see that, since $\mathfrak{A}^{\prime}$ is a center of $\mathfrak{U}$,

(1) $\mathfrak{U}^{\prime}$ satisfies (i)-(iv) above and $\mathfrak{A}^{\prime}$ satisfies all the conclusions of Theorem 1.3 with $\mathfrak{B}_{1 .}, \mathfrak{B}_{2}, \mathbb{C}_{1}, \mathfrak{C}_{2}$ held fixed.

We shall prove that, without any further use of the fact that $\mathfrak{A}^{\prime}$ is a center of $\mathfrak{A}$,

(2) there is an $\mathfrak{U}^{\prime \prime}$ (not necessarily a center of $\mathfrak{A}$ ) which satisfies (i)-(iv) above with $m$ replaced by $(m-1)$ and all the conclusions of Theorem 1.3 with $\mathfrak{B}_{1}, \mathfrak{B}_{2}, \mathbb{C}_{1}, \mathbb{C}_{2}$ held fixed.

So assume (1) and let $\mathfrak{B}_{1}^{\prime}, \mathfrak{B}_{2}^{\prime}, \mathbb{C}_{1}^{\prime}, \mathfrak{C}_{2}^{\prime}, \mathfrak{B}_{11}, \mathfrak{B}_{12}, \mathfrak{B}_{21}, \mathfrak{B}_{22}, \mathbb{C}_{11}, \mathfrak{c}_{12}, \mathbb{C}_{21}, \mathbb{C}_{22}$ be as in Theorem 1.3. Consider the factor $\mathfrak{D}_{m}$ of $\mathfrak{A}^{\prime}$. Since $\mathfrak{A}^{\prime}$ has the unique factorization property, $\mathfrak{D}_{m}$ must occur in one of $\mathfrak{B}_{1}^{\prime}$ or $\mathfrak{B}_{2}^{\prime}$ and one of $\mathbb{C}_{1}^{\prime}$ or $\mathbb{C}_{2}^{\prime}$. As we can see, there are now four cases. However, the arguments are so similar that it is sufficient to go through only one of the cases. So suppose that $\mathfrak{D}_{m}$ occurs in $\mathfrak{B}_{1}^{\prime}$ and $\mathfrak{C}_{1}^{\prime}$. Now looking at the relations in 1.3 (iv), we see that since $\mathfrak{D}_{m}$ does not occur in $\mathfrak{C}_{2}^{\prime}, \mathfrak{D}_{m}$ must occur in $\mathfrak{B}_{12}$ (this follows by an induction using (iv)). Similarly, $\mathfrak{D}_{m}$ must also occur in $\mathfrak{C}_{12}$.
So we can write

$$
\begin{aligned}
\mathfrak{B}_{1}^{\prime} \cong \mathfrak{D}_{m} \times \mathfrak{B}_{1}^{\prime \prime}, & \mathfrak{C}_{1}^{\prime} \cong \mathfrak{D}_{m} \times \mathbb{C}_{1}^{\prime \prime}, \\
\mathfrak{B}_{12} \cong \mathfrak{D}_{m} \times \mathfrak{B}_{12}^{\prime}, & \mathfrak{C}_{12} \cong \mathfrak{D}_{m} \times \mathbb{C}_{12}^{\prime} .
\end{aligned}
$$

Now, let $\mathfrak{U}^{\prime \prime}=\mathfrak{D}_{1} \times \ldots \times \mathfrak{D}_{m-1}$. Using the fact that $\mathfrak{D}_{m}$ is cancelable, we see that the structures $\mathfrak{B}_{1}^{\prime \prime}, \mathfrak{B}_{2}^{\prime}, \mathbb{C}_{1}^{\prime \prime}, \mathbb{C}_{2}^{\prime}, \mathfrak{B}_{11}, \mathfrak{B}_{12}^{\prime}, \mathfrak{B}_{21}, \mathfrak{B}_{22}, \mathfrak{C}_{11}, \mathbb{C}_{12}^{\prime}, \mathbb{C}_{21}, \mathfrak{C}_{22}$ will satisfy the conclusions of Theorem 1.3 with respect to $\mathfrak{I}^{\prime \prime}, \mathfrak{B}_{1}, \mathfrak{B}_{2}$, $\mathfrak{C}_{1}, \mathfrak{C}_{2}$. So (2) is proved from (1). Iterating this procedure, we eventually get down to the case where $m=1$. Now a refinement for $\mathfrak{B}_{1}, \mathfrak{B}_{2}, \mathfrak{C}_{1}, \mathfrak{C}_{2}$ follows from the remark after Theorem 2.1 and the proof of Theorem 2.1. The theorem is proved.

Let us see what happens to Theorem 2.2 if the indecomposable factors of $\mathfrak{U}^{\prime}$ are allowed to repeat. In the simpliest situation, suppose that $\mathfrak{H}^{\prime} \cong \mathfrak{D} \times \mathfrak{D}$, where $\mathfrak{D}$ is cancelable and indecomposable, and this representation is unique. As in the proof of 2.2 , let; $\mathfrak{A} \cong \mathfrak{B}_{1} \times \mathfrak{B}_{2} \cong \mathbb{C}_{1} \times \mathbb{C}_{2}$, and the conclusions of Theorem 1.3 hold. Suppose that it turns out that $\mathfrak{B}_{1}^{\prime} \cong \mathfrak{B}_{2}^{\prime} \cong \mathbb{C}_{1}^{\prime} \cong \mathbb{C}_{2}^{\prime} \cong \mathfrak{D}$. Then by cancelation. we have

$$
\mathfrak{B}_{i j} \cong \mathfrak{C}_{j i}, \quad i, j=1,2,
$$

whence we obtain a refinement of the four structures

$$
\mathfrak{B}_{1} \times \mathfrak{D}, \quad \mathfrak{B}_{2} \times \mathfrak{D}, \quad \mathfrak{C}_{1} \times \mathfrak{D}, \quad \mathfrak{C}_{2} \times \mathfrak{D} .
$$

Now, unless somehow we manage to find $D$ as a factor of the $\mathfrak{B}_{t j}$ 's and cancel on both sides, we apparently cannot get a refinement of $\mathfrak{B}_{1}, \mathfrak{B}_{2}$,
$\check{L}_{1}, \mathbb{C}_{2}$. This remark motivates the notion and definition of prime structures given in the introduction. It turns out that the proof of Theorem 2.8 , below, requires some preliminaries.

In the following lemmas, let $X$ be a set of prime and cancelable structures. We assume that $\mathbf{1} \in X$. So every structure in $X$ is indecomposable, except the structures isomorphic to 1 .

LEM'A 2.3. Every product of indecomposable structures from $X$ has the unique factorization property, and, whence also the refinement property.

Proof. We first prove the following proposition by induction on $n$ : Suppose that $\mathfrak{D} \in X, \mathfrak{D} \neq \mathbf{1}$, and there are indecomposable $\mathfrak{c}_{1}, \ldots, \mathfrak{C}_{n}$ such that

$$
\mathfrak{D} \| \mathbb{C}_{1} \times \ldots \times \mathfrak{C}_{n},
$$

then $\mathfrak{D} \cong \mathfrak{C}_{i}$ for some $i$. This induction is easy to carry out. Next, we prove by induction on $m$ that: if there are indecomposable $\mathfrak{D}_{1}, \ldots, \mathfrak{D}_{m} \in X$ and there are indecomposable $\mathbb{V}_{1}, \ldots, \mathbb{C}_{n}$ such that

$$
\mathfrak{D}_{1} \times \ldots \times \mathfrak{D}_{m} \cong \mathbb{C}_{1} \times \ldots \times \mathbb{C}_{n},
$$

then $m=n$ and there is a permutation $p$ of $1, \ldots, m$ such that $\mathfrak{D}_{i} \cong \mathfrak{C}_{p(i)}$. Informally, suppose that $m>1$ and (1) holds. Then

$$
\mathfrak{D}_{m} \| \mathbb{C}_{1} \times \ldots \times \mathbb{C}_{n} .
$$

So, $\mathfrak{D}_{m} \cong \mathfrak{C}_{i}$ for some $i$. Now, rearrange the $\mathbb{C}_{j}$ 's and cancel off $\mathfrak{D}_{m}$ from both sides of (1). The rest follows from the induction hypothesis. The lemma is proved.

We say that a structure $\mathfrak{B}$ is $X$-free if no structure from $X$ divides $\mathfrak{B}$.

LEMva 2.4. Every structure $\mathfrak{B}$ has a unique (up to isomorphism) representation as a product

$$
\mathfrak{B} \cong \mathfrak{B}_{1} \times \mathfrak{B}_{2}
$$

when $\mathfrak{B}_{1}$ is $X$-free and $\mathfrak{B}_{2}$ is a product of structures from $X$.

Pro of. First of all, since $\mathbf{1} \epsilon X$, we see that every structure $\mathfrak{B}$ has such a representation where $\mathfrak{B}_{1}$ is $X$-free and $\mathfrak{B}_{2}$ is a product of factors in $X$. Suppose that there are two such representations

$$
\mathfrak{B} \cong \mathfrak{B}_{1} \times \mathfrak{B}_{2} \cong \mathfrak{C}_{1} \times \mathfrak{C}_{2}
$$

We can first dismiss the trivial case where both $\mathfrak{B}_{2} \cong \mathbf{1} \cong \mathbb{C}_{2}$. By Lemma 2.3 , we may find structures $\mathfrak{C}, \mathfrak{B}_{2}^{\prime}, \mathfrak{C}_{2}^{\prime}$ all products of factors from $X$ such that

and

$$
\mathfrak{B}_{2} \cong \mathfrak{B}_{2}^{\prime} \times \mathfrak{C}, \quad \mathfrak{C}_{2} \cong \mathfrak{C}_{2}^{\prime} \times \mathfrak{C}
$$

$\mathfrak{B}_{2}^{\prime}$ and $\mathbb{C}_{2}^{\prime}$ contain no common indecomposable factors from $X$. 
Cancelling the $\mathbb{C}$ from $(1)$, we get $\mathfrak{B}_{1} \times \mathfrak{B}_{2}^{\prime} \cong \mathfrak{C}_{1} \times \mathfrak{C}_{2}^{\prime}$. If $\mathfrak{B}_{2} \not \mathfrak{C}_{2}$, then $\mathfrak{B}_{2} \neq \mathbb{C}_{2}^{\prime}$, and we may assume that there is an indecomposable $\mathfrak{D} \in X$ such that

$$
\mathfrak{D} \| \mathfrak{B}_{2}^{\prime} \text { but not } \mathfrak{D} \| \mathbb{C}_{2}^{\prime}
$$

This proves that $\mathfrak{D} \| \mathbb{C}_{1}$, which is a contradiction to the $X$-freeness of $\mathfrak{C}_{1}$. So, indeed, $\mathfrak{B}_{2} \cong \mathbb{C}_{2}$, and by cancellation, $\mathfrak{B}_{1} \cong \mathfrak{C}_{1}$.

LEMna 2.5. Suppose that $\mathfrak{B} \cong \mathfrak{B}_{1} \times \mathfrak{B}_{2}$ and $\mathfrak{C} \cong \mathfrak{C}_{1} \times \mathfrak{C}_{2}$ are the representations discussed in 2.4, then

$$
\mathfrak{B} \times \mathbb{C} \cong\left(\mathfrak{B}_{1} \times \mathfrak{C}_{1}\right) \times\left(\mathfrak{B}_{2} \times \mathfrak{C}_{2}\right)
$$

is a representation of $\mathfrak{B} \times \mathfrak{C}$.

Proof. If some indecomposable $\mathfrak{D} \in X$ divides $\mathfrak{B}_{1} \times \mathbb{C}_{1}$, then either $\mathfrak{D} \| \mathfrak{B}_{1}$ or $\mathfrak{D} \| \mathfrak{C}_{1}$, a contradiction. So $\mathfrak{B}_{1} \times \mathfrak{C}_{1}$ is $X$-free and the rest follows.

LEMViA 2.6. Suppose that $\mathfrak{A} \times \mathfrak{B} \cong \mathbb{C} \times \mathfrak{D}$, and let $\mathfrak{A}_{1}, \mathfrak{B}_{1}, \mathfrak{C}_{1}, \mathfrak{D}_{1}$ be the X-free parts of $\mathfrak{N}, \mathfrak{B}, \mathfrak{C}, \mathfrak{D}$, respectively. Then there is a refinement of $\mathfrak{A}, \mathfrak{B}, \mathfrak{C}, \mathfrak{D}$ if and only if there is a refinement of $\mathfrak{I}_{1}, \mathfrak{B}_{1}, \mathfrak{C}_{1}, \mathfrak{D}_{1}$.

Proof. We suppose that

$$
\mathfrak{U} \cong \mathfrak{I}_{1} \times \mathfrak{A}_{2}, \quad \mathfrak{B} \cong \mathfrak{B}_{1} \times \mathfrak{B}_{2}, \quad \mathfrak{C} \cong \mathfrak{C}_{1} \times \mathfrak{C}_{2}, \quad \mathfrak{D} \cong \mathfrak{D}_{1} \times \mathfrak{D}_{2},
$$

where $\mathfrak{A}_{2}, \mathfrak{B}_{2}, \mathfrak{C}_{2}, \mathfrak{D}_{2}$ are products of factors from $X$. Since $\mathfrak{A} \times \mathfrak{B}$ $\cong \mathbb{C} \times \mathfrak{D}$, we have by 2.5 and uniqueness that

and

$$
\mathfrak{U}_{1} \times \mathfrak{B}_{1} \cong \mathfrak{C}_{1} \times \mathfrak{D}_{1}
$$

$$
\mathfrak{A}_{2} \times \mathfrak{B}_{2} \cong \mathfrak{C}_{2} \times \mathfrak{D}_{2} \text {. }
$$

By Lemma 2.3, there is automatically a refinement, say $\mathbb{E}_{11}^{\prime \prime}, \mathbb{E}_{12}^{\prime \prime}, \mathbb{E}_{21}^{\prime \prime}, \mathbb{E}_{22}^{\prime \prime}$ of $\mathfrak{A}_{2}, \mathfrak{B}_{2}, \mathfrak{C}_{2}, \mathfrak{D}_{2}$. Now suppose that $\mathfrak{E}_{11}^{\prime}, \mathbb{E}_{12}^{\prime}, \mathbb{E}_{21}^{\prime}, \mathbb{E}_{22}^{\prime}$ is a refinement of $\mathfrak{A}_{1}, \mathfrak{B}_{1}, \mathfrak{C}_{1}, \mathfrak{D}_{1}$, then clearly the four structures

$$
\mathbb{E}_{11}^{\prime} \times \mathfrak{E}_{11}^{\prime \prime}, \quad \mathbb{E}_{12}^{\prime} \times \mathbb{E}_{12}^{\prime \prime}, \quad \mathfrak{E}_{21}^{\prime} \times \mathbb{E}_{21}^{\prime \prime}, \quad \mathfrak{E}_{22}^{\prime} \times \mathfrak{E}_{22}^{\prime \prime}
$$

is a refinement of $\mathfrak{A}, \mathfrak{B}, \mathfrak{C}, \mathfrak{D}$. Conversely, suppose $\mathfrak{E}_{11}, \mathfrak{E}_{12}, \mathfrak{E}_{21}, \mathfrak{E}_{22}$ is a refinement of $\mathfrak{A}, \mathfrak{B}, \mathfrak{C}, \mathfrak{D}$. We let $\mathfrak{E}_{i j}^{\prime}$ be the $X$-free partis of $\mathfrak{E}_{i j}$, respectively. A simple calculation using the uniqueness of the representation will show that

$$
\mathbb{E}_{11}^{\prime}, \mathfrak{E}_{12}^{\prime}, \mathbb{E}_{21}^{\prime}, \mathfrak{E}_{22}^{\prime} \text { is a refinement of } \mathfrak{U}_{1}, \mathfrak{B}_{1}, \mathfrak{C}_{1}, \mathfrak{D}_{1} .
$$

The lemma is proved.

LEMid 2.7. Let $\mathfrak{B}_{1}, \mathfrak{B}_{2}, \mathfrak{C}_{1}, \mathfrak{C}_{2}$ be arbitrary structures suck that $\mathfrak{B}_{1} \times \mathfrak{B}_{2}$ $\cong \mathbb{C}_{1} \times \mathbb{C}_{2}$. Let $\mathfrak{B}_{1}^{\prime}, \mathfrak{B}_{2}^{\prime}, \mathfrak{C}_{1}^{\prime}, \mathfrak{C}_{2}^{\prime}$ be products of factors from $X$. Suppose that there is a refinement of $\mathfrak{B}_{1} \times \mathfrak{B}_{1}^{\prime}, \mathfrak{B}_{2} \times \mathfrak{B}_{2}^{\prime}, \mathfrak{C}_{1} \times \mathbb{C}_{1}^{\prime}, \mathfrak{C}_{2} \times \mathbb{C}_{2}^{\prime}$. Then there is a refinement of $\mathfrak{B}_{1}, \mathfrak{B}_{2}, \mathfrak{C}_{1}, \mathfrak{C}_{2}$.
Proof. The hypothesis and Lemma $2.6 \mathrm{imply}$ that there is a refinement of the $X$-free parts of $\mathfrak{B}_{1}, \mathfrak{B}_{2}, \mathbb{C}_{1}, \mathbb{C}_{2}$, respectively. Using 2.6 once more in the opposite direction, there is a refinement of $\mathfrak{B}_{1}, \mathfrak{B}_{2}, \mathfrak{C}_{1}, \mathfrak{C}_{2}$.

THEOREM 2.8. Let $\mathfrak{H}^{\prime}$ be a center of $\mathfrak{A}$. Suppose that $\mathfrak{H}^{\prime}$ is a product of prime and cancelable factors. Then $\mathfrak{A}$ has the refinement property.

Proof. As usual suppose that $\mathfrak{A} \cong \mathfrak{B}_{1} \times \mathfrak{B}_{2} \cong \mathfrak{C}_{1} \times \mathfrak{C}_{2}$. We see that the conclusions of Theorem 1.3 hold. In particular we have, using 1.3 (ii) and $1.3(\mathrm{iv})$,

$$
\begin{aligned}
\mathfrak{B}_{1} \times \mathfrak{B}_{1}^{\prime} \times \mathbb{C}_{1}^{\prime} \times \mathbb{C}_{2}^{\prime} & \cong\left(\mathfrak{C}_{1}^{\prime} \times \mathfrak{B}_{11}\right) \times\left(\mathbb{C}_{2}^{\prime} \times \mathfrak{B}_{12}\right) \\
& \cong\left(\mathfrak{B}_{1}^{\prime} \times \mathfrak{C}_{11}\right) \times\left(\mathfrak{B}_{1} \times \mathbb{C}_{21}\right) \\
& \cong\left(\mathfrak{C}_{11} \times \mathfrak{C}_{21}\right) \times \mathfrak{B}_{1}^{\prime} \times \mathfrak{B}_{1}^{\prime} .
\end{aligned}
$$

Rewriting $\mathfrak{C}_{1}^{\prime} \times \mathfrak{C}_{2}^{\prime}$ as $\mathfrak{B}_{1}^{\prime} \times \mathfrak{B}_{2}^{\prime}$, and cancelling $\mathfrak{B}_{1}^{\prime} \times \mathfrak{B}_{1}^{\prime}$ from both sides, we have

Similarly,

$$
\mathfrak{B}_{1} \times \mathfrak{B}_{2}^{\prime} \cong \mathfrak{G}_{11} \times \mathfrak{V}_{21}
$$

$$
\mathfrak{B}_{2} \times \mathfrak{B}_{1}^{\prime} \cong \mathfrak{c}_{12} \times \mathfrak{c}_{22}
$$

So, (1), (2), and 1.3(iii) imply that there is a refinement of

$$
\mathfrak{B}_{1} \times \mathfrak{B}_{2}^{\prime}, \quad \mathfrak{B}_{2} \times \mathfrak{B}_{1}^{\prime}, \quad \mathbb{C}_{1} \times \mathfrak{C}_{1}^{\prime}, \quad \mathfrak{c}_{2} \times \mathfrak{C}_{2}^{\prime} .
$$

Now, let $X$ be the set of those prime and cancelable factors occurring in a factorization of $\mathfrak{U}^{\prime}$. Clearly $\mathfrak{B}_{2}^{\prime}, \mathfrak{B}_{1}^{\prime}, \mathfrak{C}_{1}^{\prime}, \mathfrak{C}_{2}^{\prime}$ are products of factors from $X$. Whence by Lemma 2.7 , there is a refinement of $\mathfrak{B}_{1}, \mathfrak{B}_{2}, \mathbb{C}_{1}, \mathbb{C}_{2}$. The theorem is proved.

To conclude this section, we shall restrict our attention to the class of all finite non-empty binary reflexive connected relations $T$. Let $U$ be the least equivalence relation containing $T \cap \breve{T}$, and, as before, we say that the subrelations

$$
T(U, a)=T \frown(a / U)^{2}, \quad a \in \operatorname{Fld}(T),
$$

are the centers of $T$. The preceeding three theorems can now be rephrased for connected relations $T$ as follows: (Henceforth, $E, F, G, \ldots, R, S$, $T, U, \ldots$, shall denote finite binary reflexive relations).

(1) If a center of $T$ is either isomorphic to $\{\langle 0,0\rangle\}$, or is indecomposable and cancelable (with respect to the class of relations being discussed at hand), then $T$ has the refinement property.

(2) If a center of $T$ is isomorphic to a product of distinct non-isomorphic indecomposable and cancelable factors, $U_{1} \times U_{2} \times \ldots \times U_{m}$, where

$$
U_{i} \times R \cong U_{j} \times S \text { and } i \neq j \quad \text { imply } \quad U_{i} \| S \text { and } U_{j} \| R,
$$

then $T$ has the refinement property.

Fundamenta Mathematicae, T. LX 
(3) If a center of $T$ is a product of prime and cancelable factors, then $T$ has the refinement property.

The following sequence of lemmas. is designed to prove the next theorem:

THEOREM 2.9. Let $p$ be a prime and let $p^{s}$ be the square relation $\{\langle i, j\rangle\}: 1 \leqslant i, j \leqslant p\}$. Then $p^{s}$ is cancelable and prime (among all finite binary reflexive relations).

At this point we remind the reader to look up some elementary definitions and results from [3], [2]. We would like him to recall in particular the definitions and elementary properties of

and

$$
R \cdot S, \text { the ordinal product of two relations } R, S \text {, }
$$

$$
\sum_{i, R} S_{i}, \text { the ordinal sum over } R \text { of relations } S_{i},
$$

listed, for example, on pp. 171-172 [3] and pp. 260-261 [2].

LEMINA 2.10. $p^{s}$ is cancelable.

Proof. Suppose that $p^{s} \times R \cong p^{s} \times S$. By the definitions of ordinal product of relations, we see that

$$
p^{s} \times R=p^{s} \cdot R \quad \text { and } \quad p^{s} \times S=p^{s} \cdot S .
$$

Whence we have $p^{s} \cdot R \cong p^{s} \cdot \dot{S}$. By cancelling $p^{s}$ on the left; Theorem 1 [3], we get $R \cong S$. The lemma is proved.

We say that $E$ is a congruence over $R$ if $E$ is an equivalence relation over the field of $R$ and

$$
E|R| E=R .
$$

We have already introduced the quotient relation

$$
R / E=\{\langle a \mid E, b / E\rangle: a R b\} .
$$

LeMra 2.11. If $E$ is a congruence over $R$, then

$$
R=\sum_{a \mid E, R] E}(a|E \times a| E) .
$$

Proof. This lemma depends on a straightforward verification of the definition of the ordinal sum of relations over another relation. We only need to point out that the identity $E|R| E=R$ is equivalent to the following: given $a / E$ and $b / E$,

$$
\text { if }(a \mid E \times b / E) \cap R \neq 0 \text {, then }(a / E \times b / E) \subset R .
$$

LEMMA 2.12. If $E$ and $F$ are congruences over $R$, then the least equivalence relation containing $E$ and $F$ is again a congruence over $R$.

Proof. Let $G=\bigcup_{n}\left(E \mid F^{n}\right)^{n}$. We have

$$
\left(E \mid F^{\prime}\right)|R|\left(F^{\prime} \mid E\right)=E\left|\left(F^{\prime}|R| F^{\prime}\right)\right| E=E|R| E=R .
$$

So by a simple induction we get

$$
\left(E \mid F^{n}\right)^{n}|R|(F \mid E)^{n}=R \quad \text { for every } n .
$$

This obviously implies $G|R| G=R$.

LEMMA 2.13. There is a (unique) maximal congruence relation over $R$.

Proof. Since the identity relation over the field of $R$ is a congruence over $R$, this result follows from 2.12 .

LENiv' 2.14. Suppose that $E$ is the maximal congruence over $R$ and $F$ is the maximal congruence over $S$. Then the cardinal product $G=E \times F$ is the maximal congruence over $R \times S$.

Proof. It is immediate that $G$ is an equivalence relation over the field of $R \times S$, and, furthermore,

$$
G|(R \times S)| G=R \times S .
$$

So $G$ is a congruence over $R \times S$. Suppose that $H$ is a congruence relation over $R \times S$ which properly includes $G$. We shall show that this leads to the contradiction that either $E$ or $F$ is not maximal. Say that $a, b, c, d$ are such that

$$
\langle a, b\rangle H\langle c, d\rangle \text {, but either not } a E c \text { or not } b F d .
$$

By symmetry, let us consider only the case not $a E c$. Note that since $H \subset R \times S$, we have $a R e$ and $c R a$. We define a new equivalence relation $E^{\prime}$ over the field of $R$ by

$$
E^{\prime}=E \cup(a / E \times c / E) \cup(c / E \times a / E) .
$$

$E^{\prime}$ properly includes $E$. We show that $E^{\prime}$ is a congruence over $R$. So suppose $x R y, x E^{\prime} x^{\prime}$, and $y E^{\prime} y^{\prime}$. We wish to show $x^{\prime} R y^{\prime}$. There are four cases:

(1) $x E x^{\prime}$ and $y E y^{\prime}$. This leads to $x^{\prime} R y^{\prime}$.

(2) $x\left(E^{\prime}-E\right) x^{\prime}$ and $y E y^{\prime}$. This case further divides into two subcases:

(2a) $x E a$ and $x^{\prime} E c$. So we have

$$
\langle x, b\rangle G\langle a, b\rangle H\langle c, d\rangle G\left\langle x^{\prime}, d\right\rangle \quad \text { and } \quad\langle y, b\rangle G\left\langle y^{\prime}, b\right\rangle .
$$

Since $G \subset H$, and since $\langle x, b\rangle\left(R \times S^{\prime}\right)\langle y, b\rangle$, we get $\left\langle x^{\prime}, d\right\rangle(R \times S)\left\langle y^{\prime}, b\right\rangle$. So $x^{\prime} R y^{\prime}$. 
(2b) $x E c$ and $x^{\prime} \mathbb{E} a$. We have

$$
\langle x, d\rangle G\langle c, d\rangle H\langle a, b\rangle G\left\langle x^{\prime}, b\right\rangle \quad \text { and } \quad\langle y, d\rangle G\left\langle y^{\prime}, d\right\rangle \text {. }
$$

Since $\langle x, d\rangle\left(R \times S^{\prime}\right)\langle y, d\rangle$, we have $\left\langle x^{\prime}, b\right\rangle(R \times S)\left\langle y^{\prime}, d\right\rangle$, and $x^{\prime} R y^{\prime}$.

(3) $x E x^{\prime}, y\left(\mathbb{H}^{\prime}-E\right) y^{\prime}$. Entirely analogous to case (2).

(4) $x\left(E^{\prime}-E\right) x^{\prime}$ and $y\left(E^{\prime}-E\right) y^{\prime}$. By symmetry, we consider two subcases:

(4a) $x E a, x^{\prime} E c, y E a, y^{\prime} E c$. This gives immediately $x^{\prime} E y^{\prime}$ which implies $x^{\prime} R y^{\prime}$.

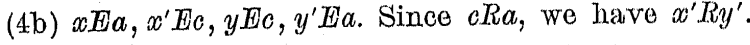

So all cases have been considered and $E^{\prime}$ is a congruence over $R$. This is a contradiction to the maximality of $t$. So $G$ must have been maximal.

We now return to the proof of Theorem 2.9 .

Proof. Suppose that $R \times p^{s} \cong S \times T$. Let $\mathbb{E}, F, G$ be the maximal congruences over $R, S, T$, respectively. Clearly, $p^{8}$ is the maximal congruence over itself. By 2.14, $E \times p^{s}$ and $F \times G$ are the maximal congruences of $R \times p^{s}$ and $S \times T$ respectively. Obviously the isomorphism carrying $R \times p^{s}$ onto $S \times T$ induces an isomorphism of $E \times p^{s}$ onto $F \times G$. So we have $E \times p^{s} \cong F \times G$. Now, each equivalence class of $E \times p^{s}$ has a number of elements which is divisible by $p$. By the isomorphism, we see that

(1) each equivalence class of $F \times G$ must have a number of elements which is divisible by $p$.

Every equivalence class of $F \times G$ is of the form

$$
a / F \times b / G, \quad a \in \operatorname{Fld}(F), \quad b \in \operatorname{Fld}(G) .
$$

So, by (1), either

$$
p \text { divides }|a| F \mid \text { for all } a \in \operatorname{Fld}\left(F^{\prime}\right),
$$

or else,

$$
p \text { divides }|b| G \mid \text { for all } b \in \operatorname{Fld}(G) \text {. }
$$

From (2), we have that $p^{s}$ divides every square relation. $a / F^{\prime} \times a / H^{\prime}$. Since,

$$
S=\sum_{a \mid F, T / F}\left(a / F \times a / F^{\prime}\right)
$$

We may factor (ordinally) $p^{s}$ from each $a / F^{\prime} \times a / F^{\prime}$. By the left distribution law of - over $\Sigma$, we may now write

$$
S \cong p^{s} \cdot S^{\prime} \cong p^{s} \times S^{\prime},
$$

so $p^{s} \| S$. In the case that (3) holds, we get $p^{s} \| T$. The theorem is proved.
We can now draw the two last consequences of this paper.

THEOREM 2.15. If $T$ is connected and a center of $T$ is a square relation, then $T$ has the refinement property.

Proof. Every (finite) square relation is a product of prime and cancelable relations of the form $p^{s}$.

THEOREM 2.16. If $T$ is connected and transitive, then $T$ has the refinement property.

Proof. In this case, the least equivalence relation containing $\breve{T} \cap T$ is simply $T \frown \breve{T}$. It is now obvious that any center of $T$ must be a square relation.

Added in proof May 23, 1967. After the submission of this manuscript, Professor Alfred Tarski brought my attention to some recent work of R. McKenzie on prime algebras (Notices of Amer. Math. Soc. 13 (1966), p. 727). While there is no overlap between our works, still $I$ think it is of some interest to point out some consequences of my results here when we restrict ourselves to the class of (finite) algebras $\mathfrak{A}=\langle A, f\rangle$ where $f$ is a binary operation, say, defined on $A$. Provided that suitable changes are made in the definitions in $\S 1$, e.g. $f(a, a)=a$ for $P(a, a, a)$, the main result Theorem 1.3 holds for algebras as well. One only has to check that if a center $\mathfrak{A}^{\prime}$ of $\mathfrak{A}$ is an algebra, then all of the structures in the conclusion of Theorem 1.3 are also algebras. The results, in $\S 2$ up to Theorem 2.8 also hold for algebras. Here, it is interesting to notice that the notions of being prime and cancelable will now be defined with respect to the class of (finite) algebras, whence they are slightly weaker notions, although the notion of being a center is, as we pointed out above, a slightly stronger notion. Thus the new Theorem 2.8 for algebras is not comparable to the old 2.8 for relational structures.

\section{References}

[1] C. C. Chang, Two theorems on direct decomposition of relations, Bull. Amer. Math. Soc. 60 (1954), p. 524.

[2] - Ordinal factorization of finite relations, Trans. Amer. Math. Soc. 101 (1961), pp. 259-293.

[3] C. C. Chang and Anne C. Morel, Some cancellation theorems for ordinal products of relations, Duke Math. J. 27 (1960), pp. 171-182.

[4] C. C. Chang, B. Jónsson, and A. Tarski, Refinement properties for relational structures, Fund. Math. 55 (1964), pp. 249-281.

[5] B. Jonsson, The unique factorization problem for finite relational structures, Colloq. Math. 14 (1966), pp. 1-32.

UNIVERSTTY OF CALIFORNIA, LOS ANGELES,

Reçu par la Rédaction le 1. 7. 1966 